\title{
Chaos in PID Controlled Nonlinear Systems
}

\begin{abstract}
Günyaz Ablay ${ }^{\dagger}$
Abstract - Controlling nonlinear systems with linear feedback control methods can lead to chaotic behaviors. Order increase in system dynamics due to integral control and control parameter variations in PID controlled nonlinear systems are studied for possible chaos regions in the closed-loop system dynamics. The Lur'e form of the feedback systems are analyzed with Routh's stability criterion and describing function analysis for chaos prediction. Several novel chaotic systems are generated from second-order nonlinear systems including the simplest continuous-time chaotic system. Analytical and numerical results are provided to verify the existence of the chaotic dynamics.
\end{abstract}

Keywords: Chaos, Feedback systems, Linear control, Nonlinear systems, Chaotification, PID control

\section{Introduction}

Chaos control and chaotification (anti-control of chaos) studies are providing significant effect on many novel applications, including high-performance circuits and devices, chemical reactions, biological systems, liquid mixing, secure communications, and critical decisionmaking in political, economic and military events [1]. Today, it seems that there are three ways to find threedimensional autonomous chaotic systems: chaotification, searching via computer programming and encountering chaos in simulations-experiments by chance [2]. Chaotification is a process that creates chaos from a non-chaotic system, or improves a chaotic system to present a different type of chaos for various potential applications in biological, medical, electronic, mechanical, optical, and chemical systems. The chaotification process usually involves designing a control mechanism. Various chaos control methods, e.g. OGY method [3], delayed feedback control $[4,5]$, washout filter $[6,7]$, periodic perturbations [8-10], stochastic perturbations [11], and nonlinear control methods [12] can be used to alter dynamics of chaotic systems, but in this study chaotification means generating a chaotic structure from a non-chaotic system.

In recent years, some studies have been devoted to chaotification in discrete-time and continuous-time dynamical systems. Most of these studies have been done in the endeavor of achieving chaotification in discrete maps, and have showed that chaotification in discrete-time systems is very promising [13-19]. On the other hand, for continuous-time dynamical systems, some feedback control based studies recently have been available in the literature with some successful works, including timedelayed feedback and impulsive control approaches [2035]. The chaotification process is different in discrete-time and continuous-time systems since their dynamic properties

$\dagger$ Corresponding Author: Dept. of Electrical and Electronic Engineering, Abdullah Gül University, Turkey. (gunyaz.ablay@agu.edu.tr) Received: November 20, 2014; Accepted: February 23, 2015 are completely different, e.g. a discrete chaotic system can have all positive Lyapunov exponents but a continuous chaotic system needs only a positive Lyapunov exponent to achieve stretch and fold mechanism. It is a common way to use trial-error approach through parameter tuning and numerical simulation in order to get chaotic systems [17, $25,36]$. In this work, the common classical control tools, i.e. PID control, Routh's stability criterion and describing function analysis, will be used for chaotification in a systematic way.

The main goal of the study is to investigate and show that chaotic dynamics can occur in PID controlled continuous-time nonlinear systems. PID controller is the most common control approach used in industry [37]. It is also an effective method for controlling chaos [38-40] and chaos synchronization [41, 42]. On the other hand, variations in the PID control parameters can result in generation of chaos from a non-chaotic nonlinear system. Since the integral term of the PID controller increases order of a system, it is possible to produce chaotic systems from second-order autonomous nonlinear systems. In this way, some new chaotic systems, including the simplest continuous-time chaotic system, are presented in this paper. These new chaotic systems pose high potentials for various applications in certain scientific, engineering and industrial fields, e.g. secure communications and circuit designs.

The paper is organized as follows. Section 2 presents novel chaotic dynamics under PID controlled nonlinear systems, and conclusion of the work is given in Section 3.

\section{Chaos in Feedback Systems}

Feedback control systems are the most common and successful approaches for solving practical control problems. On the other hand, controller parameters can be adjusted in such a way that unexpected behaviors including bifurcations and chaos can occur. Consider a second-order nonlinear system described by 


$$
\ddot{y}+a \dot{y}+b y+f(y, \dot{y}, u)=u
$$

where $a$ and $b$ are the system parameters, the function $f(y, \dot{y}, u)$ represents the nonlinear part of the system and $u$ is a feedback controller. Let the feedback controller be [37]

$$
u=-k_{1} \dot{y}-k_{2} y-k_{3} \sigma, \quad \dot{\sigma}=y
$$

where $k_{1}, k_{2}$ and $k_{3}$ are the controller gains. By substituting the controller (2) into the system (1) and introducing new state variables as $x=\sigma, \dot{x}=y$ and $\ddot{x}=\dot{y}$, we get

$$
\dddot{x}+k_{d} \ddot{x}+k_{p} \dot{x}+k_{i} x=g(x, \dot{x}, \ddot{x})
$$

where the new system parameters are $k_{d}=k_{1}+a$, $k_{p}=k_{2}+b$ and $k_{i}=k_{3}$, and the function $g(\cdot)$ is the nonlinear term of the feedback system. The system (3) can be represented by the combination of the linear term $G(\cdot)$ and the nonlinear term $g(\cdot)$, known as Lur'e form depicted in Fig. 1. Note that the nonlinear parts of the chaotic systems to be generated in this work will depend on the system output $(\dot{x}=y)$, while many other nonlinear terms can also be considered (e.g., system (10)).

The system (3) can exhibit chaotic behaviors depending on its parameters and initial conditions. Chaotic systems include an infinite number of unstable periodic orbits embedded in an ergodic chaotic attractor [3]. This means that a chaotic motion is a type of limit cycle which is perturbed by the stretching and folding mechanism. By considering the process of periodic-doubling route to chaos, occurrence of a stable limit cycle is an important sign to chaotic behavior. Existence of such a limit cycle in nonlinear systems can be predicted with the describing function analysis that is a powerful tool for analyzing nonlinear systems [43]. The condition for the existence of a limit cycle is given by

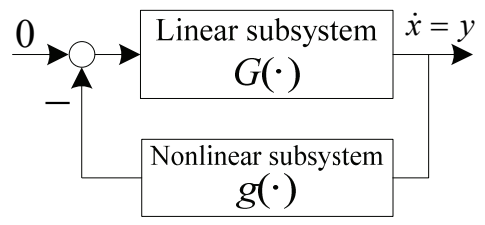

Fig. 1. A feedback system in the Lur'e form.

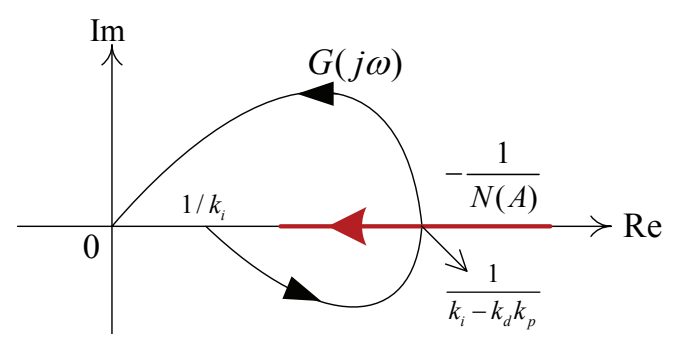

Fig. 2. Nyquist plot for stable limit cycle prediction.

$$
G(j \omega)=-1 / N(A)
$$

where $N(\cdot)$ is the describing function of the nonlinear term. Eq. (4) states that each intersection point of the curve $G(j \omega)$ and the trajectory of $-1 / N(A)$ corresponds to a limit cycle, which is illustrated in Fig. 2. A specific method for analyzing chaotic systems in Lur'e form is based on describing function analysis proposed by Genesio and Tesi [44]. Genesio-Tesi procedure [45] for determining chaotic behavior of a system in Lur'e form necessitates: (a) Existence of an interacting stable limit cycle and unstable fixed point, and (b) suitable filtering effect along the system. This procedure gives approximate necessary conditions for the existence of a homoclinic orbit, whose perturbation can result in chaos $[45,46]$.

A fast and effective way for finding existence of an interacting stable limit cycle and unstable fixed point can be obtained by using Routh's stability condition and Nyquist plot of the linear subsystem $G(\cdot)$. In the presence of the nonlinear term $g(\cdot)$, if a stable limit cycle or chaotic behavior exists for the system (3), then

(i) $k_{d}, k_{i}>0$ and $k_{i}>k_{d} k_{p}$,

(ii) describing function $N(\cdot)$ of the nonlinear term $g(\cdot)$ must intersect with the Nyquist plot of linear term $G(\cdot)$ as depicted in Fig. 2.

Besides, periodic solutions exist if $k_{i}=k_{d} k_{p}$. The suitable filtering effect along the system has a qualitative and heuristic nature, but this can easily be solved with numerical simulations. That is to say, after achieving conditions (i) and (ii), a rough bifurcation diagram of the system (3) can be obtained for a selected range of parameters in order to search for the existence of chaos. In the following subsections, system (3) will be evaluated for various nonlinearities for the existence of chaos with the approach outlined above.

\subsection{Quadratic nonlinearities}

For a quadratic nonlinearity, $g= \pm \dot{x}^{2}$, the system (3) can be rewritten as

$$
\dddot{x}+k_{d} \ddot{x}+k_{p} \dot{x}+k_{i} x= \pm \dot{x}^{2}
$$

The system (6) has a fixed point at $x_{e}=(0,0,0)$, and linearization at the fixed point results in the characteristic equation $s^{3}+k_{d} s^{2}+k_{p} s+k_{i}=0$. When the system parameters are selected as $k_{d}=2.6, k_{p}=0.1$ and $k_{i}=1.81$, it can easily be shown that unstable fixed point (condition (i)) and stable limit cycle (condition (ii)) conditions are satisfied for the possibility of chaos. A bifurcation diagram exhibiting a period-doubling route to chaos for the system (6) is shown in Fig. 3 for the parameter $k_{i}$ versus maximum value of $x$. The numerical simulations are done using MATLAB programs. It is clear 


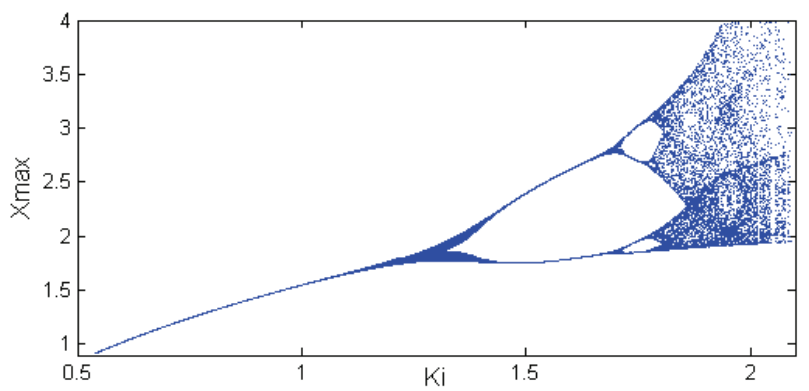

Fig. 3. A bifurcation diagram for the quadratic system (6) for the parameter $k_{i}$ versus maximum value of $x$.
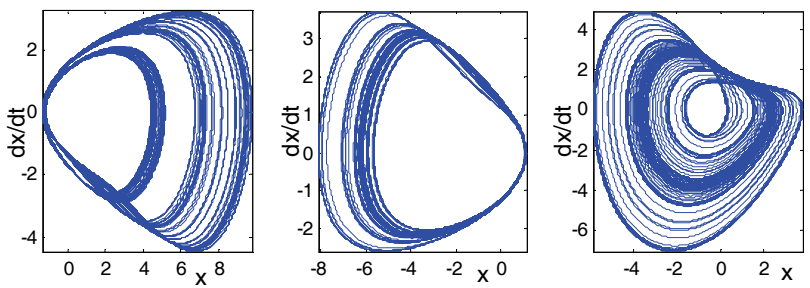

Fig. 4. Chaotic attractors for quadratic nonlinearities: (a) system (6), (b) system (9) and (c) system (10).

that there is an interleaving of chaos and order for $1.8 \leq k_{i} \leq 2.1$. Fig. 4(a) shows the attractor of this new chaotic system.

For further assessment of this new chaotic system, eigenvalues of the system are calculated as

$$
s_{1}=-2.79, \quad s_{2,3} \equiv \sigma \pm j \omega=0.098 \pm 0.79 i
$$

Hence, the fixed point $x_{e}$ is a saddle focus and satisfies the inequality $\left|s_{1}\right|>|\sigma|>0$, which implies that there is a homoclinic chaos according to Shil'nikov theorem [47-49]. In addition, the chaotic attractor has a dissipative feature since the divergence of flows is described by

$$
\partial \dddot{x} / \partial \ddot{x}=-k_{d}<0
$$

Eq. (8) shows that all system orbits will be confined to a specific limit set of zero volume and asymptotic motion will converge to an attractor [50]. The dimension of the attractor can be estimated through Kaplan-Yorke dimension as $D_{K Y}=2.0076$ (see Table 2).

It is interesting to see that when $k_{p}=0$ in (6), a one of the simplest chaotic system (five-term) can be obtained. Namely,

$$
\dddot{x}+k_{d} \ddot{x}+k_{i} x=-\dot{x}^{2}
$$

where $k_{d}=2.38$ and $k_{i}=1.6$. The system (9) has a fixed point $x_{e}=(0,0,0)$, and linearization at the fixed point yields three eigenvalues, $s_{1}=-2.61$ and $s_{2,3}=$ $0.11 \pm 0.77 i$. Again, the fixed point $x_{e}$ is a saddle focus

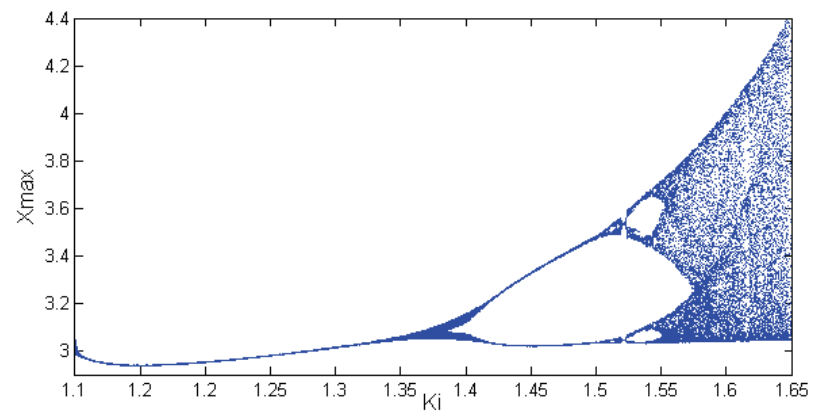

Fig. 5. Bifurcation diagram of the simplest chaotic system (9) for $k_{i}$ versus maximum value of the $x$.

and satisfies the inequality $\left|s_{1}\right|>|\sigma|>0$. Consequently, the system produces a homoclinic chaos. The chaotic attractor has a dissipative feature because $\partial \dddot{x} / \partial \ddot{x}=-k_{d}<0$. System (9) is one of the simplest chaotic systems, since it has been proved that four-term autonomous systems cannot exhibit chaos [51]. While there are some other simple five-term chaotic systems (including one quadratic term [52] and two quadratic terms [53]), this new chaotic system has a much larger chaos region (i.e., the existence of chaos in certain ranges of parameter variations, e.g. $1.55 \leq k_{i} \leq 1.65$ ) compared to the chaotic dynamic introduced in [52].

Fig. 4(b) shows the attractor of the chaotic system (9). A bifurcation diagram of the system (9) for the parameter $k_{p}$ versus maximum value of $x$ exhibiting a period-doubling route to chaos is presented in Fig. 5. Complex solutions exist for $k_{d}=2.38$ and $1.1 \leq k_{i} \leq 1.65$. The dimension of the attractor is $D_{K Y}=2.0179$, but the basin of attractor is small and initial conditions must be chosen carefully.

The quadratic nonlinearity can also be considered as a product of two different state variables. When the nonlinearity is described by $g(\cdot)= \pm x \dot{x}$, another simple dissipative chaotic system can be introduced by

$$
\dddot{x}+k_{d} \ddot{x}+k_{p} \dot{x}+k_{i} x= \pm x \dot{x}
$$

where the system parameters are $k_{d}=2.6, k_{p}=0.53$ and $k_{i}=3.8$. The bifurcation diagram of the system (10) for $k_{i}$ versus $x_{\max }$ is presented in Fig. 6. It is seen that chaotic solutions exist for $3.5<k_{i}<4.5$. The fixed point of the system is at the origin and carries the features of the

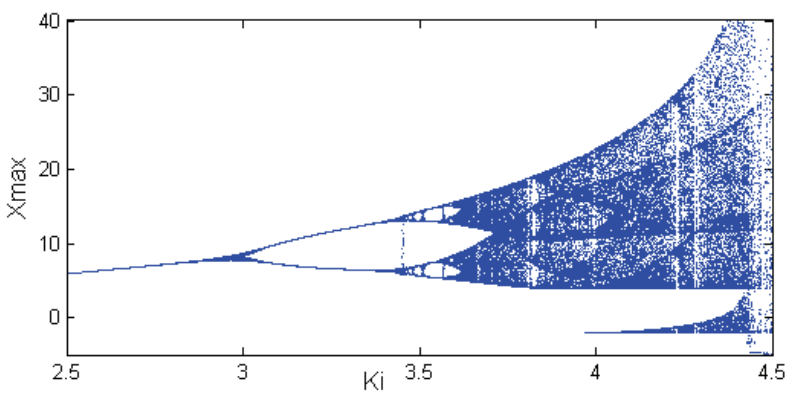

Fig. 6. Bifurcation diagram of the system (10) for $k_{i}$ versus maximum value of $x$. 
saddle focus since the eigenvalues are $s_{1}=-2.87$ and $s_{2,3} \equiv \sigma \pm j \omega=0.13 \pm 1.14 i$. A homoclinic chaos exists since $\left|s_{1}\right|>|\sigma|>0$. Fig. 4(c) shows the attractor of this novel chaotic system.

\subsection{Cubic/quintic nonlinearities}

The nonlinear function of the system (3) can be composed of a simple cubic nonlinearity,

$$
\dddot{x}+k_{d} \ddot{x}+k_{p} \dot{x}+k_{i} x=-\dot{x}^{3}
$$

where the system parameters are $k_{d}=0.9, k_{p}=-9$ and $k_{i}=5$. System (11) has a fixed point $x_{e}=(0,0,0)$, which is a saddle point. The unstable fixed point and stable limit cycle conditions (conditions (i) and (ii)) are satisfied for the possibility of chaos. For $3.6 \leq k_{i} \leq 5.1$, a perioddoubling route to chaos scheme can be observed in the numerical simulations and the bifurcation diagram given in Fig. 8. The existence of the chaotic attractor (Fig. 7a) can be given by $\partial \ddot{x} / \partial \ddot{x}=-k_{d}<0$ and observed in numerical simulations. Since divergence of flows is negative, the system (11) is dissipative and the dimension of the attractor is calculated as $D_{K Y}=2.0526$.

A more complicated form of the cubic nonlinearity, known as odd quartic function, can also yield a chaotic behavior,

$$
\dddot{x}+k_{d} \ddot{x}+k_{p} \dot{x}+k_{i} x=-\dot{x}^{3}|\dot{x}|
$$

where the system parameters are $k_{d}=1, k_{p}=-5$ and $k_{i}=4$. For the possibility of chaos, conditions (i) and (ii) are satisfied, and a bifurcation diagram of the system
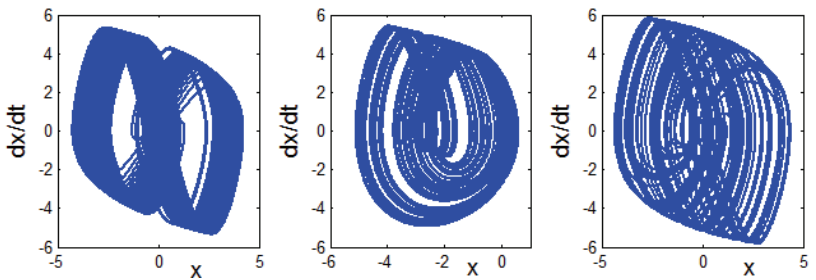

Fig. 7. Chaotic attractors for various nonlinearities, (a) cubic (11), (b) exponential (14), (c) hyperbolic (15).

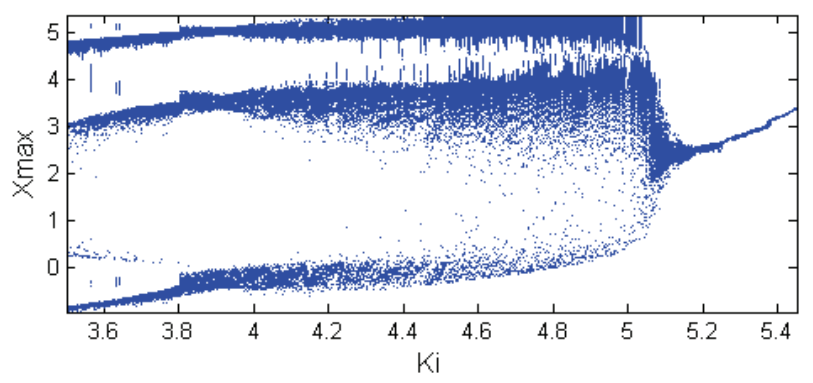

Fig. 8. Bifurcation diagram of the cubic system (11) for $k_{i}$ versus maximum value of $x$. exhibiting a period-doubling route to chaos is displayed in Fig. 9. It is seen form the bifurcation diagram that there is a wide chaos region $\left(2.5 \leq k_{i} \leq 5.1\right)$, but the continuity of this region is disrupted with periodic windows. Moreover, there is only a single fixed point $x_{e}=(0,0,0)$, and it is a saddle focus since its eigenvalues are calculated as $s_{1}=-3.06$ and $s_{2,3} \equiv \sigma \pm j \omega=1.03 \pm 0.49 i$. This system also produces a homoclinic chaos since $\left|s_{1}\right|>|\sigma|>0$. The divergence of flows is $\partial \ddot{x} / \partial \ddot{x}=-k_{d}<0$ and thus, the system is dissipative. The dimension of the attractor is $D_{K Y}=2.1228$.

Another dissipative chaotic system example can be obtained with a quintic nonlinearity,

$$
\dddot{x}+k_{d} \ddot{x}+k_{p} \dot{x}+k_{i} x=-\dot{x}^{5}
$$

where the system parameters are $k_{d}=1, k_{p}=-9$ and $k_{i}=10$. The bifurcation diagram of the system (13) is shown in Fig. 10. Similar to the other chaotic systems, this system also has a saddle fixed point $x_{e}=(0,0,0)$, and exhibits a homoclinic chaos. The dimension of the attractor is calculated as $D_{K Y}=2.0754$ and Lyapunov exponents of the system are given in Table 2. Compared to the other chaotic systems introduced in this section, the bifurcation diagram in Fig. 10 shows that the system (13) has the largest chaos region $\left(5.5 \leq k_{i} \leq 16\right)$.

\subsection{Exponential and hyperbolic nonlinearities}

The physical systems can also have exponential,

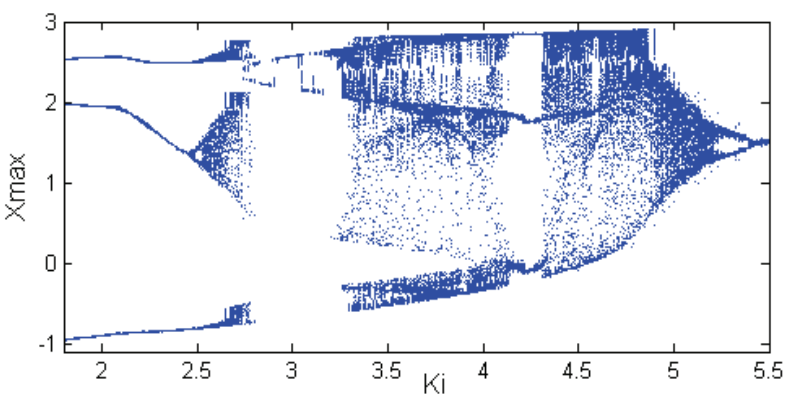

Fig. 9. Bifurcation diagram of the system (12) for $k_{i}$ versus maximum value of $x$.

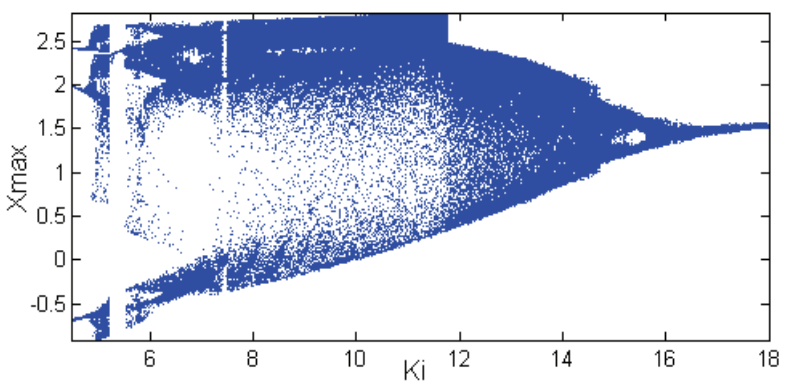

Fig. 10. Bifurcation diagram of the quintic system (13) for $k_{i}$ versus maximum value of $x$. 
hyperbolic and trigonometric nonlinearities. By considering a saturated exponential function, the reference system (3) becomes

$$
\dddot{x}+k_{d} \ddot{x}+k_{p} \dot{x}+k_{i} x=1-\exp (\dot{x})
$$

where the system parameters are $k_{d}=1, k_{p}=-1$ and $k_{i}=7$. A bifurcation diagram of the system exhibiting a period-doubling route to chaos is depicted in Fig. 11. There is an interleaving chaos and order for $0 \leq k_{i} \leq 8.2$, interrupted with some periodic windows. The fixed point of the system (14) is at the origin and behaves as a saddle focus, and the system exhibits a homoclinic chaos. The system has a dissipative characteristics because $\partial \dddot{x} / \partial \ddot{x}=-k_{d}<0$, and the dimension of the attractor is found as $D_{K Y}=2.1304$. The attractor is shown in Fig. $7 \mathrm{~b}$.

Now, let the system (3) contain a hyperbolic function,

$$
\dddot{x}+k_{d} \ddot{x}+k_{p} \dot{x}+k_{i} x=-\sinh (\dot{x})
$$

where the system parameters are $k_{d}=1, k_{p}=-5$ and $k_{i}=5$. The bifurcation diagram of the system is illustrated in Fig. 12. Similar to the other chaotic systems, the system (15) has a saddle fixed point $x_{e}=(0,0,0)$ and exhibits a homoclinic chaos. The dimension of the attractor of this dissipative chaotic system is calculated as $D_{K Y}=2.1667$. The attractor is displayed in Fig. 7c.

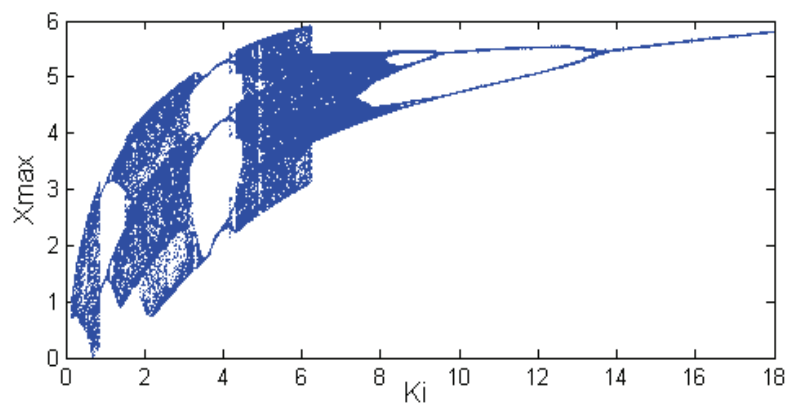

Fig. 11. Bifurcation diagram of the exponential system (14) for $k_{i}$ versus maximum value of $x$.

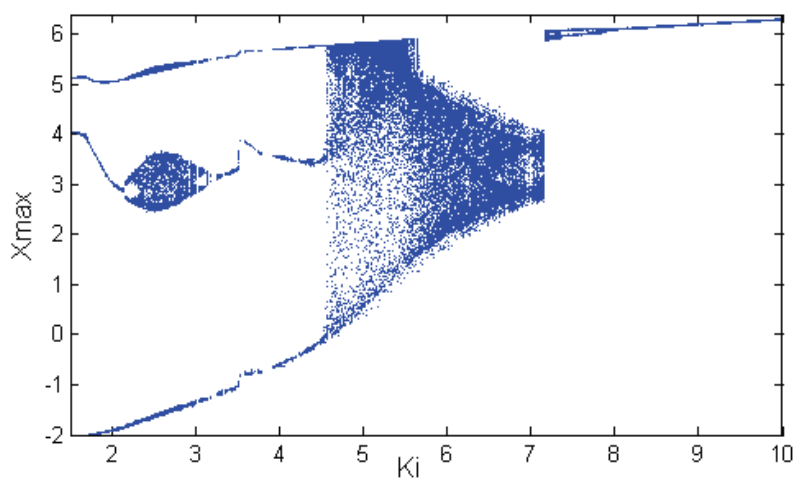

Fig. 12. Bifurcation diagram of the hyperbolic system (15) for $k_{i}$ versus maximum value of $x$

\subsection{Characterization of the novel chaotic systems}

The describing function and stable limit cycle prediction of some common nonlinear functions are provided in Table 1. It is seen from Table 1 that it is not always possible to get stable limit cycle prediction for all nonlinearities. In addition, while some nonlinearities can yield a stable limit cycle, they may not produce a chaotic behavior under system (3). It is also interesting to see that the common hard nonlinearities, e.g. $\operatorname{sign}(\cdot)$ function, cannot yield a stable limit cycle and chaotic behavior by considering the system (3).

The characterization of the attractors of chaotic systems is an important aspect. The geometry of chaotic attractors can be characterized with the dimension of an attractor. One significant quantitative characterization method is the Kaplan-Yorke dimension (KYD) [54]. It represents an upper bound for the information dimension of the system. The KYD is calculated by $D_{K Y}=d+\left(\lambda_{1}+\ldots+\lambda_{d}\right) /\left|\lambda_{d+1}\right|$ where $\lambda_{1} \leq \lambda_{d}$ are the Lyapunov exponents (LEs) and $d$ is

Table 1. Analysis of common nonlinearities and chaos

\begin{tabular}{|c|c|c|c|}
\hline $\begin{array}{c}\text { Nonlinear } \\
\text { function }\end{array}$ & $\begin{array}{l}\text { Describing } \\
\text { function }\end{array}$ & Graphical analysis & Comments \\
\hline$-y|y|$ & $-8 A / 3 \pi$ & & $\begin{array}{l}\text { Stable limit cycle, } \\
\text { apparently no } \\
\text { chaos }\end{array}$ \\
\hline$-y^{2}$ & $-2 A$ & & $\begin{array}{l}\text { Stable limit cycle } \\
\text { and chaos }\end{array}$ \\
\hline$-y^{3}$ & $-3 A^{2} / 4$ & & $\begin{array}{l}\text { Stable limit cycle } \\
\text { and chaos }\end{array}$ \\
\hline$-y^{3}|y|$ & $-32 A^{3} / 15 \pi$ & & $\begin{array}{l}\text { Stable limit cycle } \\
\text { and chaos }\end{array}$ \\
\hline$-\sqrt{y}$ & $-1.11 / \sqrt{A}$ & & $\begin{array}{l}\text { Unstable limit } \\
\text { cycle, no chaos }\end{array}$ \\
\hline$-\operatorname{sign}(y)$ & $-4 / A \pi$ & & $\begin{array}{l}\text { Unstable limit } \\
\text { cycle, no chaos }\end{array}$ \\
\hline$-\sinh (y)$ & $-2 \mathrm{I}_{1}(A) / A$ & & $\begin{array}{l}\text { Stable limit cycle } \\
\text { and chaos }\end{array}$ \\
\hline $1-e^{y}$ & $\frac{2}{A}\left[\mathrm{I}_{1}(A)+\mathrm{S}_{1}(A)\right]$ & & $\begin{array}{c}\text { Stable limit cycle } \\
\text { and chaos }\end{array}$ \\
\hline$-y^{2} \dot{y}$ & $-A^{2} j \omega / 4$ & 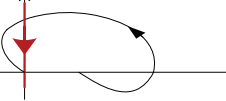 & $\begin{array}{c}\text { Stable limit cycle, } \\
\text { apparently no } \\
\text { chaos }\end{array}$ \\
\hline
\end{tabular}
prediction for the system (3).

$\mathrm{I}_{1}(A)$ and $\mathrm{S}_{1}(A)$ are the modified Bessel and Struve functions of order 1 , respectively. 
Table 2. Novel chaotic dynamics in feedback systems.

\begin{tabular}{c|c|c|c}
\hline Novel chaotic systems & LEs & KSE & KYD \\
\hline$\dddot{x}+2.6 \ddot{x}+0.1 \dot{x}+1.81 x= \pm \dot{x}^{2}$ & $(0.02,0,-2.62)$ & 0.02 & 2.0076 \\
\hline$\dddot{x}+2.38 \ddot{x}+1.6 x=-\dot{x}^{2}$ & $(0.043,0,-2.42)$ & 0.043 & 2.0179 \\
\hline$\dddot{x}+2.6 \ddot{x}+0.53 \dot{x}+3.8 x= \pm x \dot{x}$ & $(0.053,0,-2.65)$ & 0.053 & 2.0206 \\
\hline$\dddot{x}+0.9 \ddot{x}-9 \dot{x}+5 x=-\dot{x}^{3}$ & $(0.05,0,-0.95)$ & 0.05 & 2.0526 \\
\hline$\dddot{x}+\ddot{x}-9 \dot{x}+10 x=-\dot{x}^{5}$ & $(0.08,0,-1.06)$ & 0.08 & 2.0754 \\
\hline$\dddot{x}+\ddot{x}-5 \dot{x}+4 x=-\dot{x}^{3}|\dot{x}|$ & $(0.14,0,-1.14)$ & 0.14 & 2.1228 \\
\hline$\dddot{x}+\ddot{x}-\dot{x}+7 x=1-\exp (\dot{x})$ & $(0.15,0,-1.15)$ & 0.15 & 2.1304 \\
\hline$\dddot{x}+\ddot{x}-5 \dot{x}+5 x=-\sinh (\dot{x})$ & $(0.2,0,-1.2)$ & 0.2 & 2.1667 \\
\hline & & &
\end{tabular}

the largest integer for which $\lambda_{1}+\ldots+\lambda_{d} \geq 0$. For the calculation of LEs, the Wolf algorithm [55] is used. It is seen from Table 2 that LEs of the introduced chaotic systems consist of positive, zero and negative exponents. Since the chaotic systems are dissipative, the sum of the LEs is negative. LEs can also be used for unpredictability measure of the chaotic dynamics, i.e. Kolmogolov-Sinai entropy (KSE) [56] calculations. KSE is defined by the sum of the positive LEs, and gives information about the level of the randomness of a chaotic system. The greater KSE means the higher randomness. Table 2 summarizes the LEs, KSE and KYD of the novel continuous-time chaotic systems introduced in this work.

\section{Conclusion}

This paper presented novel chaotic systems generated from PID controlled second-order nonlinear systems. The study set out to determine the possibility of chaotic behaviors in simple nonlinear systems controlled with PID systems. This research has shown that the integral control term of the PID controller increases system order and makes chaotification possible in second-order systems with simple nonlinearities. The chaos prediction approach is based on the Routh's stability criterion and describing function analysis. Several new chaotic systems, including the simplest chaotic dynamic, are introduced, and their existences are shown with bifurcation diagrams and Lyapunov exponents. These novel chaotic systems have high potentials in many applications including secure communications, circuit designs and random number generations. This research also shows that bifurcations and chaos can occur in industrial systems regulated by the PID controllers.

\section{Acknowledgements}

This work was supported by Research Fund of the Abdullah Gül University. Project No: 4.

\section{References}

[1] G. Chen, "Chaos, Bifurcations, and Their Control," in Wiley Encyclopedia of Electrical and Electronics Engineering, John Wiley \& Sons, Inc., 2001.

[2] J. Lü, G. Chen, and D. Cheng, "A new chaotic system and beyond: The generalized Lorenz-like system," Int. J. Bifurcation Chaos, vol. 14, no. 05, pp. 1507-1537, May 2004.

[3] E. Ott, C. Grebogi, and J. A. Yorke, "Controlling chaos," Physical Review Letters, vol. 64, no. 11, pp. 1196-1199, 1990.

[4] K. Pyragas, "Delayed feedback control of chaos," Philos Trans Roy Soc A, vol. 364, pp. 2309-2334, 2006.

[5] K. Pyragas, "Continuous control of chaos by selfcontrolling feedback," Physics Letters A, vol. 170, no. 6, pp. 421-428, Nov. 1992.

[6] A. Tesi, E. H. Abed, R. Genesio, and H. O. Wang, "Harmonic balance analysis of period-doubling bifurcations with implications for control of nonlinear dynamics," Automatica, vol. 32, no. 9, pp. 1255-1271, Sep. 1996.

[7] S. Zhou, X. Lin, and H. Li, "Chaotic synchronization of a fractional-order system based on washout filter control," Comm. in Nonlinear Science and Numerical Simulation, vol. 16, no. 3, pp. 1533-1540, 2011.

[8] Y. Braiman and I. Goldhirsch, "Taming chaotic dynamics with weak periodic perturbations," Phys. Rev. Lett., vol. 66, no. 20, pp. 2545-2548, 1991.

[9] Y. Lei, W. Xu, Y. Xu, and T. Fang, "Chaos control by harmonic excitation with proper random phase," Chaos, Solitons \& Fractals, vol. 21, no. 5, pp. 11751181, Sep. 2004.

[10] R. Li, W. Xu, and S. Li, "Chaos controlling of extended nonlinear Liénard system based on the Melnikov theory," Applied Mathematics and Computation, vol. 178, no. 2, pp. 405-414, 2006.

[11] S. Fahy and D. R. Hamann, "Transition from chaotic to nonchaotic behavior in randomly driven systems," Phys. Rev. Lett., vol. 69, no. 5, pp. 761-764, 1992.

[12] G. Ablay, "Sliding mode control of uncertain unified chaotic systems," Nonlinear Analysis: Hybrid Systems, vol. 3, no. 4, pp. 531-535, 2009.

[13] X. F. Wang and G. Chen, "On feedback anticontrol of discrete chaos," International Journal of Bifurcation and Chaos, vol. 09, no. 07, pp. 1435-1441, Jul. 1999.

[14] S. Wiggins, Introduction to applied nonlinear dynamical systems and chaos. New York: Springer, 2003.

[15] G. Chen and Y. Shi, "Introduction to anti-control of discrete chaos: theory and applications," Philos Trans A Math Phys Eng Sci, vol. 364, no. 1846, pp. 24332447, Sep. 2006.

[16] Y. Shi and G. Chen, "Chaotification of discrete dynamical systems governed by continuous maps," 
International Journal of Bifurcation and Chaos, vol. 15 , no. 02, pp. 547-555, Feb. 2005.

[17] G. Hu, "Generating hyperchaotic attractors with three positive lyapunov exponents via state feedback control," International Journal of Bifurcation and Chaos, vol. 19, no. 02, pp. 651-660, Feb. 2009.

[18] Z. Li, J.-B. Park, and Y.-H. Joo, "Chaotifying continuous-time TS fuzzy systems via discretization," IEEE Transactions on Circuits and Systems I: Fundamental Theory and Applications, vol. 48, no. 10, pp. 1237-1243, Oct. 2001.

[19] S. Codreanu, "Desynchronization and chaotification of nonlinear dynamical systems," Chaos, Solitons \& Fractals, vol. 13, no. 4, pp. 839-843, Mar. 2002.

[20] J. Zhang, D. Xu, J. Zhou, and Y. Li, "Chaotification of vibration isolation floating raft system via nonlinear time-delay feedback control," Chaos, Solitons \& Fractals, vol. 45, no. 9-10, pp. 1255-1265, Sep. 2012.

[21] A. S. Kuznetsov and S. P. Kuznetsov, "Parametric generation of robust chaos with time-delayed feedback and modulated pump source," Comm. in Nonlinear Science and Numerical Simulation, vol. 18, no. 3, pp. 728-734, 2013.

[22] S. Yu and G. Chen, "Chaotifying continuous-time nonlinear autonomous systems," Int. Journal of Bifurcation and Chaos, vol. 22, no. 09, p. 12, 2012.

[23] S. Sahin and C. Guzelis, "Chaotification of Real Systems by Dynamic State Feedback," IEEE Antennas and Propagation Magazine, vol. 52, no. 6, pp. 222233, Dec. 2010.

[24] Q. Xie, Z. Han, and W. Zhang, "Chaotification via system immersion," Journal of Computational and Applied Mathematics, vol. 236, no. 7, pp. 1775-1782, Jan. 2012.

[25] X. F. Wang, "Generating Chaos in Continuous-Time Systems via Feedback Control," in Chaos Control, G. Chen and X. Yu, Eds. Springer Berlin Heidelberg, 2003, pp. 179-204.

[26] H.-K. Chen and C.-I. Lee, "Anti-control of chaos in rigid body motion," Chaos, Solitons \& Fractals, vol. 21, no. 4, pp. 957-965, Aug. 2004.

[27] Z.-M. Ge, C.-M. Chang, and Y.-S. Chen, "Anticontrol of chaos of single time scale brushless dc motors and chaos synchronization of different order systems," Chaos, Solitons \& Fractals, vol. 27, no. 5, pp. 1298-1315, Mar. 2006.

[28] M. U. Akhmet and M. O. Fen, "Replication of chaos," Comm. in Nonlinear Science and Numerical Simulation, vol. 18, no. 10, pp. 2626-2666, 2013.

[29] S. Raiesdana and S. M. H. Goplayegani, "Study on chaos anti-control for hippocampal models of epilepsy," Neurocomputing, vol. 111, pp. 54-69, Jul. 2013.

[30] P. Saha, S. Banerjee, and A. R. Chowdhury, "On the study of control and anti-control in magneto-con- vection," Physics Letters A, vol. 306, no. 4, pp. 211226, Jan. 2003.

[31] J. Moreno-Valenzuela, "Adaptive anti control of chaos for robot manipulators with experimental evaluations," Comm. in Nonlinear Science and Numerical Simulation, vol. 18, no. 1, pp. 1-11, 2013.

[32] Y. Li, W. K. S. Tang, and G. Chen, "Hyperchaos evolved from the generalized Lorenz equation," International Journal of Circuit Theory and Applications, vol. 33, no. 4, pp. 235 - 251, 2005.

[33] J. Zhou, D. Xu, J. Zhang, and C. Liu, "Spectrum optimization-based chaotification using time-delay feedback control," Chaos, Solitons \& Fractals, vol. 45, no. 6, pp. 815-824, Jun. 2012.

[34] D. O. T. Fossi and P. Woafo, "Generation of complex phenomena in a simple electromechanical system using the feedback control," Communications in Nonlinear Science and Numerical Simulation, vol. 18, no. 1, pp. 209-218, Jan. 2013.

[35] K. Starkov and G. Chen, "Chaotification of polynomial continuous-time systems and rational normal forms," Chaos, Solitons \& Fractals, vol. 22, no. 4, pp. 849-856, Nov. 2004.

[36] S. Yu and G. Chen, "Anti-control of continuous-time dynamical systems," Communications in Nonlinear Science and Numerical Simulation, vol. 17, no. 6, pp. 2617-2627, Jun. 2012.

[37] K. J. Åström and T. Hägglund, Advanced PID Control. ISA, 2006.

[38] S. Das, A. Acharya, and I. Pan, "Simulation studies on the design of optimum PID controllers to suppress chaotic oscillations in a family of Lorenz-like multiwing attractors," Mathematics and Computers in Simulation, vol.100, pp. 72-87, 2014.

[39] A. Alfi, "Chaos suppression on a class of uncertain nonlinear chaotic systems using an optimal $\mathrm{H} \infty$ adaptive PID controller," Chaos, Solitons \& Fractals, vol. 45, no. 3, pp. 351-357, Mar. 2012.

[40] D. Davendra, I. Zelinka, and R. Senkerik, "Chaos driven evolutionary algorithms for the task of PID control," Computers \& Mathematics with Applications, vol. 60, no. 4, pp. 1088-1104, 2010.

[41] L. dos S. Coelho and R. B. Grebogi, "Chaotic synchronization using PID control combined with population based incremental learning algorithm," Expert Systems with Applications, vol. 37, no. 7, pp. 5347-5352, Jul. 2010.

[42] C.-F. Hsu, J.-Z. Tsai, and C.-J. Chiu, "Chaos synchronization of nonlinear gyros using self-learning PID control approach," Applied Soft Computing, vol. 12, no. 1, pp. 430-439, Jan. 2012.

[43] D. Atherton, An Introduction to Nonlinearity in Control Systems. Bookboon, 2011.

[44] R. Genesio and A. Tesi, "Chaos prediction in nonlinear feedback systems," Control Theory and Applications, IEE Proceedings D, vol. 138, no. 4, pp. 
313-320, Jul. 1991.

[45] R. Genesio and A. Tesi, "Harmonic balance methods for the analysis of chaotic dynamics in nonlinear systems," Automatica, vol. 28, no. 3, pp. 531-548, May 1992.

[46] J. Guckenheimer and P. Holmes, Nonlinear oscillations, dynamical systems, and bifurcation of vector fields. New York: Springer-Verlag, 1983.

[47] D. V. Turaev and L. P. Shilnikov, "An example of a wild strange attractor," Sbornik Mathematics, vol. 189, no. 2, pp. 291-314, 1998.

[48] L.P. Shilnikov, "A case of the existence of a denumerable set of periodic motions," Sov. Math. Dokl., vol. 6, pp. 163-166, 1965.

[49] C. P. Silva, "Shil'nikov's theorem-a tutorial," IEEE Trans Circuits and Systems I: Fundamental Theory and Applications, vol. 40, no. 10, pp. 675-682, 1993.

[50] B. R. Hunt, The Theory of Chaotic Attractors. Springer, 2004.

[51] Z. Fu and J. Heidel, "Non-chaotic behaviour in threedimensional quadratic systems," Nonlinearity, vol. 12, no. 3, p. 739, May 1999.

[52] J. C. Sprott, "Simple chaotic systems and circuits," American Journal of Physics, vol. 68, no. 8, pp. 758763, 2000.

[53] B. Munmuangsaen and B. Srisuchinwong, "A new five-term simple chaotic attractor," Physics Letters A, vol. 373, no. 44, pp. 4038-4043, Oct. 2009.

[54] P. Frederickson, J. L. Kaplan, E. D. Yorke, and J. A. Yorke, "The liapunov dimension of strange attractors," Journal of Differential Equations, vol. 49, no. 2, pp. 185-207, Aug. 1983.

[55] A. Wolf, J. B. Swift, H. L. Swinney, and J. A. Vastano, "Determining Lyapunov exponents from a time series," Physica D: Nonlinear Phenomena, vol. 16, no. 3, pp. 285-317, Jul. 1985.

[56] R. Frigg, "In what sense is the Kolmogorov-Sinai Entropy a measure for chaotic behaviour? Bridging the gap between dynamical systems theory and communication theory," British J. for the Philosophy of Science, vol. 55, no. 3, pp. 411-434, 2004.

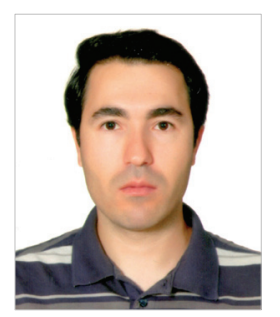

Günyaz Ablay He received his $\mathrm{PhD}$ degree in nuclear engineering from the Ohio State University, and his MS and BS degrees in electrical engineering from Firat University. His research interests include control theory and its applications, chaos theory and nuclear energy technologies. 